\title{
Wave Propagation In Subexcitable Media With Periodically Modulated Excitability
}

Irene Sendiña-Nadal

Eugene Mihaliuk

Jichang Wang

Vicente Pérez-Muñuzuri

Kenneth Showalter

Follow this and additional works at: https://researchrepository.wvu.edu/faculty_publications

\section{Digital Commons Citation}

Sendiña-Nadal, Irene; Mihaliuk, Eugene; Wang, Jichang; Pérez-Muñuzuri, Vicente; and Showalter, Kenneth, "Wave Propagation In Subexcitable Media With Periodically Modulated Excitability" (2001). Faculty Scholarship. 732.

https://researchrepository.wvu.edu/faculty_publications/732 


\title{
Wave Propagation in Subexcitable Media with Periodically Modulated Excitability
}

\author{
Irene Sendiña-Nadal, ${ }^{1}$ Eugene Mihaliuk, ${ }^{2}$ Jichang Wang, ${ }^{2}$ Vicente Pérez-Muñuzuri, ${ }^{1}$ and Kenneth Showalter ${ }^{2, *}$ \\ ${ }^{1}$ Group of Nonlinear Physics, Facultade de Físicas, Universidade de Santiago de Compostela, 15706 Santiago de Compostela, Spain \\ ${ }^{2}$ Department of Chemistry, West Virginia University, Morgantown, West Virginia 26506-6045
}

(Received 24 April 2000; revised manuscript received 2 October 2000)

\begin{abstract}
Wave propagation in a photosensitive, subexcitable Belousov-Zhabotinsky medium is made possible by periodic modulation of a homogeneous illumination field. The propagation can be understood in terms of an interplay between the radial expansion of the wave and the motion of its free ends as the excitability varies periodically. This description leads to a simple kinematic analysis that provides insights into the initial conditions and forcing parameters giving rise to sustained wave propagation.
\end{abstract}

DOI: 10.1103/PhysRevLett.86.1646

PACS numbers: $82.40 . \mathrm{Bj}, 47.35 .+\mathrm{i}, 47.54 .+\mathrm{r}$

Spatially extended dynamical systems display interesting and sometimes unexpected behavior in response to external perturbations $[1,2]$. Temporal perturbations of excitable media [3-7], as well as spatial modulations [8-10] and spatiotemporal fluctuations [11-14], give rise to new types of dynamical behavior. In this Letter, we study the effects of spatially homogeneous, periodic modulations of excitability on wave propagation in a subexcitable medium. We distinguish between three different levels of excitability of a medium: excitable, unexcitable, and subexcitable. In excitable media, waves initiated by perturbations of sufficient magnitude (amplitude and spatial size [15]) propagate throughout a continuous medium. In unexcitable media, any initial perturbation simply decays until it disappears. The subexcitable regime lies between these two excitability levels. For excitabilities below the first level, wave segments with free ends either expand or contract, depending on their size and the medium excitability. Unconditional propagation failure (even for unbounded waves) defines the second level, below which the medium is unexcitable [16].

In our experiments, the medium is prepared in the subexcitable regime and we then modulate the excitability around this reference state. We find, for certain amplitudes and frequencies of excitability modulation, that propagating wave segments do not disappear over time as they do without the modulation. We present experimental examples of modulation supported wave propagation and corroborate these results with numerical studies. We also offer a simple kinematic analysis.

Experiments were carried out with the photosensitive Belousov-Zhabotinsky (BZ) reaction $[17,18]$, which has proven to be an ideal model system for studies of perturbed excitable media. The ruthenium catalyst of the reaction, when photochemically excited with $460 \mathrm{~nm}$ light, reacts with bromomalonic acid to produce bromide [19], which is an inhibitor of autocatalysis. The excitability of the medium can therefore be controlled by varying the light intensity. In order to maintain the chemical medium in a particular subexcitable state, the light intensity was set to a reference value, $I_{0}$. A modulation of the excitability was then introduced by varying the light intensity as a square wave, with values $I_{0} \pm A / 2$ alternating with period $T$.

Panel (a) of Fig. 1 shows overlaid images taken at equal time intervals of a wave propagating in the subexcitable medium (corresponding to constant illumination at the reference intensity $I_{0}$ ). We see the contraction and collapse of the wave segment due to a negative tangential component of the velocity at the free ends of the wave. Panel 1(b) shows that the propagation distance of the wave is increased with the application of a periodic modulation of the light intensity around $I_{0}$. With longer modulation periods, the wave propagation is greatly enhanced, as shown in panel 1(c). Sustained wave propagation is exhibited for certain periods and amplitudes of light intensity modulation that generate excitabilities above and below the subexcitable reference state on an appropriate time scale.

A two-variable Oregonator model [20,21], modified to include the photosensitivity of the $\mathrm{BZ}$ reaction [22], provides insights into the support of wave propagation by periodic modulation of excitability:

$$
\begin{aligned}
& \frac{\partial u}{\partial t}=\frac{1}{\varepsilon}\left(u-u^{2}-(f v+\phi) \frac{u-q}{u+q}\right)+D_{u} \nabla^{2} u, \\
& \frac{\partial v}{\partial t}=(u-v),
\end{aligned}
$$

where the dimensionless variables $u$ and $v$ correspond to $\mathrm{HBrO}_{2}$ and the $\mathrm{Ru}(\mathrm{III})$ catalyst concentrations, respectively, and $D_{u}=1$ is the scaled diffusion coefficient. Since the $\mathrm{Ru}(\mathrm{III})$ is bound to the silica gel medium, there is no corresponding diffusion term for $v$. The parameter $\phi$ gives the light-induced production of $\mathrm{Br}^{-}$, which is directly proportional to the intensity of the illumination.

Figure 2 shows the evolution of wave fronts calculated with Eq. (1) for different values of the modulation period $T$ and a constant amplitude $A$. Wave propagation in the subexcitable medium without periodic modulation is shown in panel 2(a). With $T$ less than a critical value, the temporal modulation of excitability temporarily prolongs the life of the wave front, as shown in panel 2(b), but does not prevent its eventual collapse. Sustained wave propagation occurs at the critical period $T_{c}$, shown in panel 2(c), 


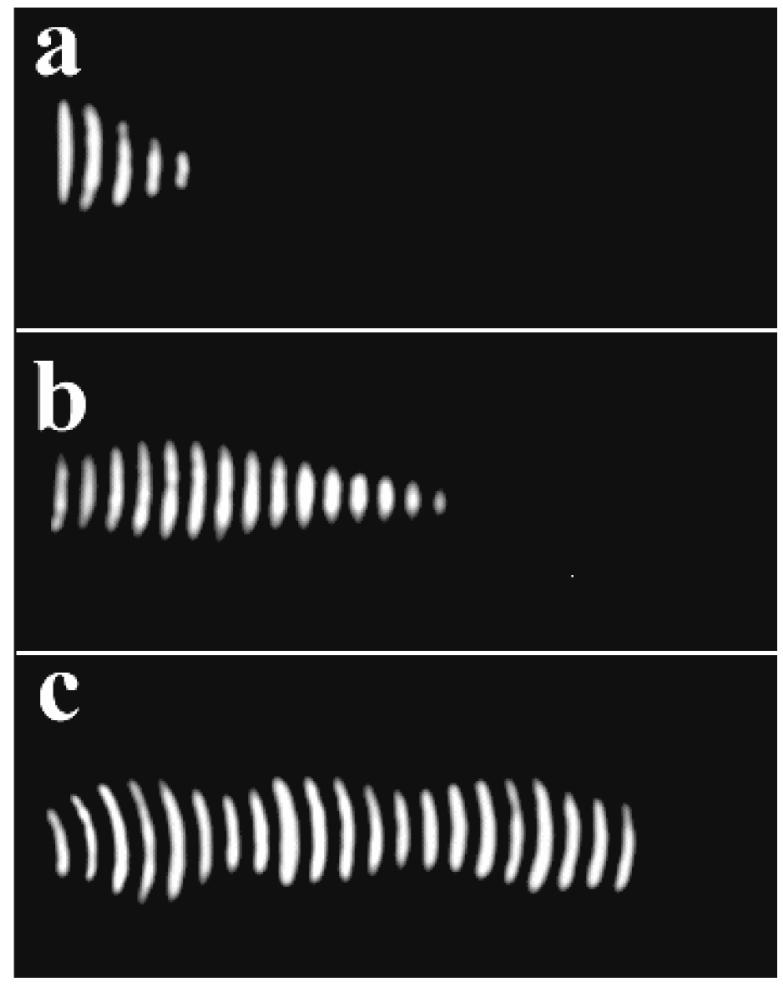

FIG. 1. Overlay of images taken every $20 \mathrm{~s}$ of a wave propagating in a subexcitable light-sensitive $\mathrm{BZ}$ medium with periodic modulation of light intensity: $I=I_{0} \pm A / 2$, $I_{0}=3.87 \mathrm{~mW} / \mathrm{cm}^{2}$, and $A=0.22 \mathrm{~mW} / \mathrm{cm}^{2}$. (a) Wave evolution with no modulation, (b) partial support of wave propagation with a modulation period of $T=66 \mathrm{~s}$, and (c) sustained propagation with $T=133 \mathrm{~s}$. The reaction takes place in an open reactor, continuously supplied with fresh, catalyst-free $\mathrm{BZ}$ solution $(0.27 \mathrm{M} \mathrm{NaBrO}, 0.05 \mathrm{M}$ malonic acid, $0.2 \mathrm{M} \mathrm{H}_{2} \mathrm{SO}_{4}, 0.15 \mathrm{M}$ bromomalonic acid) in order to maintain a nonequilibrium state. The catalyst, ruthenium (II)-bipyridyl, was immobilized in a silica gel matrix $0.3 \mathrm{~mm}$ thick [15\% w/w Na $\mathrm{Na}_{2} \mathrm{SiO}_{3} 2 \mathrm{mM} \mathrm{Ru}(\text { bpy })_{3}^{2+}$, recipe as in [25]], cast on a $2 \mathrm{~cm} \times 3 \mathrm{~cm}$ microscope slide [26]. The medium domain is $1.59 \mathrm{~cm} \times 0.74 \mathrm{~cm}$. A video projector served as a computer controlled light source, with a center wavelength of $\lambda=460 \mathrm{~nm}$ selected by means of a bandpass interference filter $\Delta \lambda=10 \mathrm{~nm}$. A video camera was used to record the evolution of the patterns and to monitor the homogeneity of the modulation.

where the wave front displays a periodic modulation in length corresponding to the periodic modulation in excitability. For periods longer than $T_{c}$, the wave front grows until it reaches the boundary of the medium, panel 2(d). Once pinned at the boundaries, the wave takes on the features of an unbounded planar wave, which propagates indefinitely for these conditions.

The effects of periodic excitability modulation on wave propagation are summarized in the phase diagram shown in Fig. 3, which shows the qualitative asymptotic behavior as a function of the forcing period and amplitude for a given set of initial conditions. Along the critical line, a balance between the rates of contraction and expansion gives rise

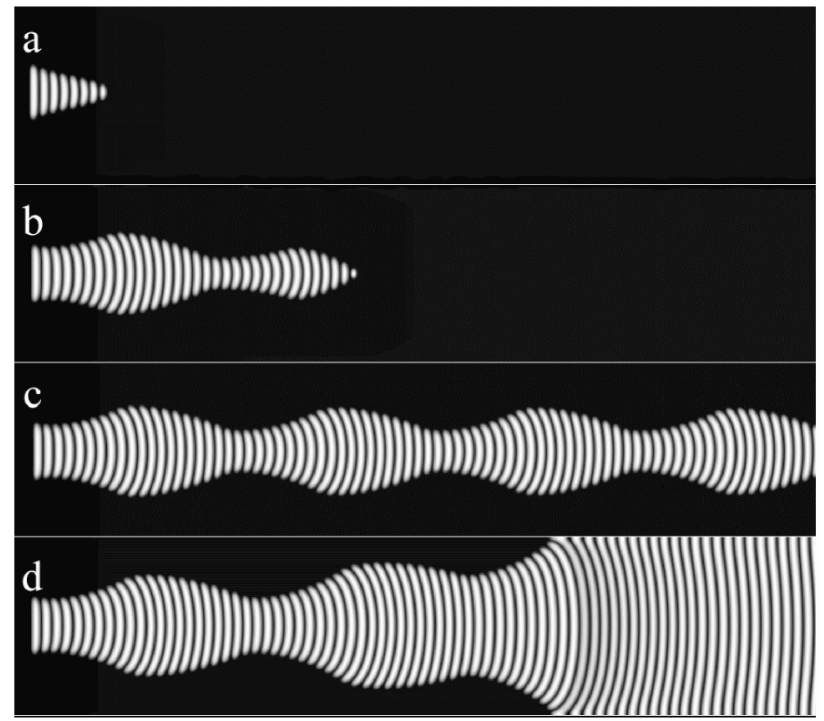

FIG. 2. Calculated wave behavior using the Oregonator model of the photosensitive BZ reaction, Eq. (1). Excitability is modulated periodically through $\phi=\phi_{0} \pm A / 2$, where $\phi_{0}=0.0766$ and $A=0.004$, as a square wave with period $T$. (a) Wave evolution with no modulation, (b) partial support of wave propagation with a modulation period $T=9$, (c) sustained propagation with $T=10$, and (d) expanding wave with $T=11$. The overlays are composed of snapshots taken every 0.5 time units. Equation (1) was integrated using an Euler method with zero flux boundary conditions and a five point Laplacian operator in an array of $900 \times 200$ points (time step $10^{-3}$ and grid point spacing 0.15 ). The parameters were $\varepsilon=0.03, f=1.4$, and $q=0.002$; the initial length of the wave segment was the same in all calculations (ca. 6.8)

to sustained wave propagation. Below this line, the modulation is not sufficient to prevent wave collapse, although wave propagation is increasingly enhanced for periods and amplitudes approaching the line. Above the critical line, the excitability modulation supports propagation, and the wave segment increases in length as it propagates. In our experiments and model calculations, this expansion always resulted in an extension of the wave to the boundaries of the rectangular reaction domain. For conditions close to the critical line, the wave was sufficiently far from the medium boundary during the first few modulation periods $T$ that the width of the medium did not play any role. The role of the initial phase of modulation is discussed below in association with Fig. 4.

Chemical wave propagation in a subexcitable medium can be analyzed using a simple kinematic model for the motion of a curved wave front with free ends [23,24]. For every value of excitability in the subexcitable regime there exists a propagating wave with a particular constant size and shape. The curvature of such wave segments increases from the middle of the segment to the ends. As the middle regions of the wave expand outward to take the place of the ends that disappear due to the negative tangential velocity, the curvature gradually increases with the decreasing normal velocity along the front, thus preserving the constant 


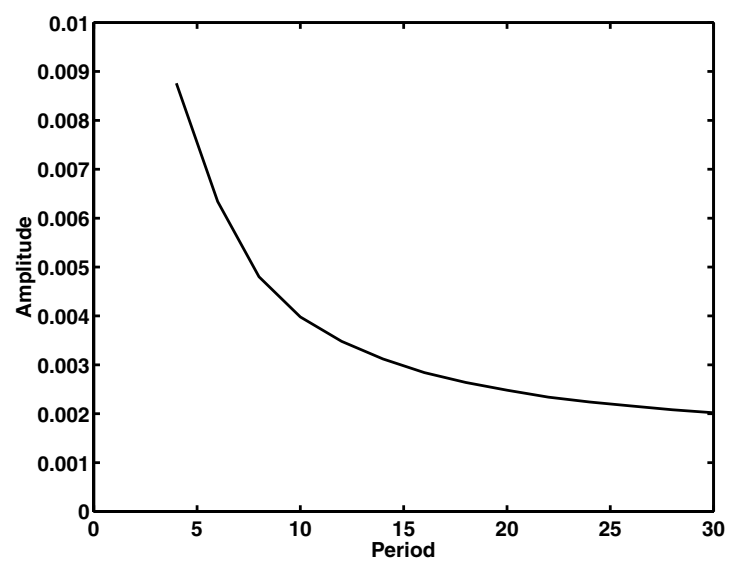

FIG. 3. Wave behavior of Eq. (1) for different values of modulation period and amplitude. Parameter values below the line correspond to decaying wave fronts; parameter values above the line correspond to expanding wave fronts. The boundary corresponds to sustained propagation of wave fronts with periodically modulated length. System parameters and numerical integration are the same as in Fig. 2.

profile. The stability of this solution is dependent upon the balance of radial expansion and tangential shrinking of the wave segment. For small deviations from the steady state shape, the rate of the length change is proportional to the deviation, giving rise to an exponential growth or decay:

$$
\ell(t)=\left(\ell_{0}-\ell_{c}\right) e^{t / \tau}+\ell_{c}
$$

where $\ell_{0}=\ell(0)$ is the initial length, $\tau$ is the characteristic time, and $\ell_{c}$ is the critical length, such that when $\ell_{0}>\ell_{c}$, $\ell(t) \rightarrow \infty$ and when $\ell_{0}<\ell_{c}, \ell(t) \rightarrow 0$ for $t \rightarrow \infty$. We note that Eq. (2) holds only for small deviations from the

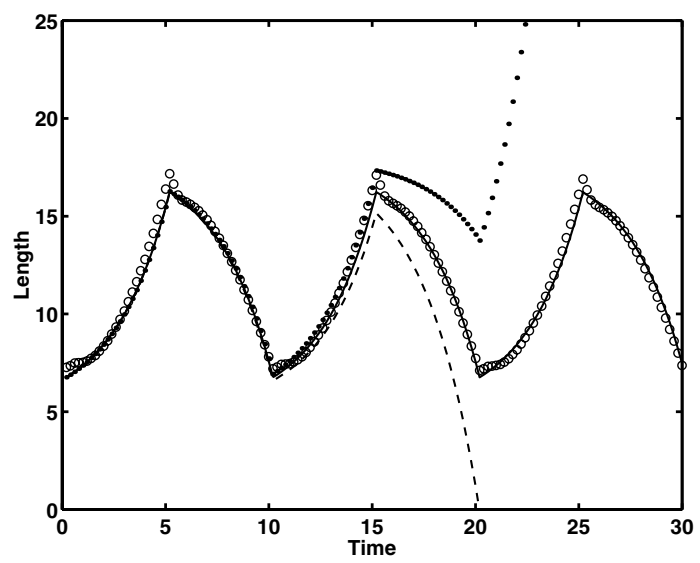

FIG. 4. Half-length of expanding (dotted line), sustained (solid line), and contracting (dashed line) waves according to Eq. (3). Modulation periods are $T=9.985,10.000$, and 10.015, respectively, with 10.000 being the critical period for the inital half-length $l_{0}=6.66$. Parameters are $l_{c}^{-}=5, l_{c}^{+}=18$, and $\tau^{+}=\tau^{-}=2.6$. Circles correspond to length of wave calculated from Eq. (1) with the same parameters as in Fig. 2, panel (c). unstable steady state length; however, it may serve as a useful approximation in a wider range of conditions.

Observations suggest that modulation of excitability results in two effectively separate modes of wave evolution, each approximately described by its own set of empirical kinematic parameters that can be measured from images of propagating wave fronts. These parameters define two critical lengths $\ell_{c}^{-}<\ell_{c}^{+}$and the corresponding time constants $\tau^{-}$and $\tau^{+}$. The solution for the modulated medium can be written as a piecewise function:

$\ell(t)= \begin{cases}\left(\ell_{0}-\ell_{c}^{-}\right) e^{t / \tau^{-}}+\ell_{c}^{-}, & 0<t \leq \frac{T}{2}, \\ {\left[\ell\left(\frac{T}{2}\right)-\ell_{c}^{+}\right] e^{[t-(T / 2)]\left(1 / \tau^{+}\right)}+\ell_{c}^{+},} & \frac{T}{2}<t \leq T,\end{cases}$

where $t$ is taken modulo $T$. Since the length changes periodically in time, the length at the end of the period must return to its initial value, $\ell(T)=\ell(0)$, which leads to the following relation between the initial condition $l_{0}$ and the forcing parameters:

$$
\ell_{0}=\frac{\ell_{c}^{-}\left(1-e^{T / 2 \tau^{-}}\right) e^{T / 2 \tau^{+}}+\ell_{c}^{+}\left(1-e^{T / 2 \tau^{+}}\right)}{1-e^{(T / 2)\left[\left(1 / \tau^{-}\right)+\left(1 / \tau^{+}\right)\right]}} .
$$

Figure 4 shows the evolution of the front length given by Eq. (3). The parameters are chosen to approximate the wave behavior in Fig. 2. The three trajectories correspond to three different modulation periods. The modulation with the critical period $T_{c}$, found from Eq. (4) for this particular initial length, results in a periodic solution. The modulation with a lower period, $T<T_{c}$, produces a decaying solution; the modulation with a higher period, $T>T_{c}$, produces an unconstrained growth in the length of the wave. Figure 4 shows that the kinematic description predicts behavior that is qualitatively similar to the predictions based on Eq. (1) for the wave at the critical forcing period.

We have not considered the initial phase of the modulation as a parameter in the above discussion; however, its effect is demonstrated by the periodic solution in Fig. 4. In this context, the solution can also be viewed as the critical initial length for different initial modulation phases. Hence, for any initial phase, at a corresponding starting point on the curve, the evolution continues along the periodic solution. Any initial state above the solution diverges to infinity, and any initial state below the solution vanishes.

The support of wave propagation in a subexcitable medium by excitability modulation can be understood by considering the wave segment evolution during each half of the driving period. During the half when the medium is more excitable, the wave develops a curved shape and increases in length; during the half when the medium is less excitable, the wave shrinks and becomes quasiplanar. Both the amplitude and period of the modulation, as well as the initial length and curvature of the wave segment, determine which process dominates and, hence, whether the wave shrinks and disappears or grows and continues to 
propagate. When these processes are balanced, sustained propagation is exhibited, as shown in panel (c) of Fig. 2.

In summary, we have found that periodic modulation of excitability around a subexcitable state may give rise to sustained wave propagation, depending on the forcing parameters and initial conditions. For critical values of these constraints, the sustained propagation assumes a form where the length of the wave varies periodically with the period of the external modulation.

This study was carried out during a research visit by I. S.-N. to West Virginia University. K. S. thanks the National Science Foundation (CHE-9974336) and the Office of Naval Research for supporting this research. This work was partially supported by the Comisión Interministerial de Ciencia y Tecnología (DGES) and Consellería de Educación e Ordenación Universitaria (Xunta de Galicia) under Projects No. PB97-0540 and No. XUGA20602B97. We thank Michael Hildebrand for useful discussions.

*Corresponding author.

Email address: kshowalt@wvu.edu

[1] M. C. Cross and P. C. Hohenberg, Rev. Mod. Phys. 65, 851 (1993).

[2] Chemical Waves and Patterns, edited by R. Kapral and K. Showalter (Kluwer Academic, Dordrecht, 1993).

[3] K. I. Agladze, V. A. Davydov, and A.S. Mikhailov, JETP Lett. 45, 767 (1987).

[4] V.S. Zykov, O. Steinbock, and S. C. Müller, Chaos 4, 509 (1994).

[5] O. Steinbock, V. Zykov, and S. C. Müller, Nature (London) 366, 322 (1993).

[6] V. Petrov, Q. Ouyang, and H. L. Swinney, Nature (London) 388, 655 (1997).

[7] A. P. Muñuzuri, M. Gómez-Gesteira, V. Perez-Muñuzuri, V. I. Krinsky, and V. Pérez-Villar, Phys. Rev. E 50, 4258 (1994).

[8] M. Markus, Zh. Nagy-Ungvarai, and B. Hess, Science 257, 225 (1992).

[9] V. G. Fast and A. M. Pertsov, J. Cardiovasc. Electrophysiol. 3, 255 (1992).
[10] A. P. Muñuzuri, C. Innocenti, J.-M. Flesselles, J.-M. Gilli, K. I. Agladze, and V. I. Krinsky, Phys. Rev. E 50, 667 (1994).

[11] I. Sendiña-Nadal, S. Alonso, V. Pérez-Muñuzuri, M. Gómez-Gesteira, V. Pérez-Villar, L. Ramírez-Piscina, J. Casademunt, J. M. Sancho, and F. Sagués, Phys. Rev. Lett. 84, 2734 (2000).

[12] V. Pérez-Muñuzuri, F. Sagués, and J. M. Sancho, Phys. Rev. E 62, 94 (2000).

[13] S. Kádár, J. Wang, and K. Showalter, Nature (London) 391, 770 (1998).

[14] J. Wang, S. Kádár, P. Jung, and K. Showalter, Phys. Rev. Lett. 82, 855 (1999).

[15] Á. Tóth, V. Gáspár, and K. Showalter, J. Phys. Chem. 98, 522 (1994).

[16] The tangential velocity changes from positive to negative at the first excitability level and the normal velocity becomes zero at the second excitability level. The subexcitable regime can be further subdivided into two regions: a region at higher excitability, where waves with free ends either expand or contract depending on their size and the particular excitability, and a region at lower excitability, where all waves with free ends contract and eventually disappear.

[17] A. N. Zaikin and A. M. Zhabotinsky, Nature (London) 225, 535 (1970).

[18] L. Kuhnert, Nature (London) 319, 393 (1986).

[19] S. Kádár, T. Amemiya, and K. Showalter, J. Phys. Chem. 101, 8200 (1997).

[20] R. J. Field and R. M. Noyes, J. Chem. Phys. 60, 1877 (1973).

[21] J. J. Tyson and P. C. Fife, J. Chem. Phys. 73, 2224 (1980).

[22] H.-J. Krug, L. Pohlmann, and L. Kuhnert, J. Phys. Chem. 94, 4862 (1990).

[23] A. S. Mikhailov, Foundations of Synergetics I: Distributed Active Systems (Springer, Berlin, 1990).

[24] A. S. Davydov, V. S. Zykov, and A. S. Mikhailov, Sov. Phys. Usp. 34, 665 (1991); Physica (Amsterdam) 70D, 1 (1994).

[25] T. Yamaguchi, L. Kuhnert, Zs. Nagy-Ungvaray, S.C. Müller, and B. Hess, J. Phys. Chem. 95, 5831 (1991).

[26] T. Amemiya, S. Kádár, P. Kettunen, and K. Showalter, Phys. Rev. Lett. 77, 3244 (1996). 